\title{
Research on the Significance and Path of Building a Government Ruled by Law Under the Background of Big Data
}

\begin{abstract}
Yu Weitao ${ }^{1, \text { a }}$
${ }^{1}$ School of Law and Humanities and Social Sciences, Wuhan University of Technology, Wuhan, Hubei, China alittle_lawson0929@163.com

ABSTRACT

Since the 18th National Congress of the Communist Party of China, the construction of a government under the rule of law, as an important part of the modernization of national governance capabilities and governance system, is an important part of deepening the "two centenary" goals and realizing the "Chinese Dream". The 21 st century is an information age. By using big data to help the construction of a government under the rule of law, it will help expand the sources of government information, enhance the accuracy of construction evaluation, and improve the efficiency of government services, thereby forming a long-term improvement and guarantee mechanism, which is effective for the rule of law. Government construction plays an active role in promoting.
\end{abstract}

Keywords: big data, government's legal construction, social governance

\section{大数据背景下法治政府建设的意义与路径探究}

\author{
余为韬 $1, a^{*}$ \\ 1 武汉理工大学法学与人文社会学院, 武汉, 湖北, 中国 \\ alittle_lawson0929@163.com \\ 摘要: \\ 党的十八大以来，法治政府建设作为国家治理能力与治理体系现代化的重要内容，是深入推进 “两个百年” 奋 \\ 斗目标，实现 “中国梦” 的重要组成部分。二十一世纪是信息化时代，通过使用大数据助力法治政府建设，有 \\ 助于扩大政务信息来源、增强建设评估准确性、提高政府服务的效能，进而形成长效的改进与保障机制，对法 \\ 治政府建设有着积极地促进作用。
}

关键词: 大数据 政府法治化建设 社会治理

\section{1. 概述}

中共十九届四中全会于 2019 年 10 月 28 日在北 京召开, 会议审议并通过了《关于坚持和完善中国特 色社会主义制度、推进国家治理体系和治理能力现代 化若干重大问题的决定》(以下简称《决定》) 这一重 要文件。《决定》在开篇中强调: “坚持全面依法治国, 建设社会主义法治国家, 切实保障社会公平正义和人
民权利的显著优势”，同时明确要求 “坚持完善中国 特色社会主义法治体系，提高党依法治国、依法执政 能力”。如何扎实推进依法治国、努力提升国家治理 体系及治理能力现代化的难题不仅是各级领导干部， 同时也是每一位法学学者及法律事务工作者所应当 面对的。 


\section{2. 法治政府建设的核心问题说}

法治政府建设作为依法治国的重要组成部分, 是 国家治理体系现代化的基石, 其建设的重要意义不言 而喻。法治政府建设的核心问题有以下几个方面:

\section{1. 坚持党的领导}

“坚持中国特色社会主义法治道路,最根本的是 坚持中国共产党的领导。” 这是习总书记在十八届四 中全会上所做的重要发言。法治政府作为法治国家的 主体, 自然也应当坚持党的领导。因为行政立法、行 政执法、行政争议解决的事务并非单由行政机关内部 决定, 而是涉及到行政权力运用的方方面面, 单靠行 政机关是无法满足法治政府建设更深层次的变革, 需 要有党的领导作指引。十八大以后成立的全面依法治 国委员会进一步强化了党在科学立法、公正司法、严 格执法、全民守法中的领导作用, 统筹推进全面依法 治国建设; 目前开展的法治政府建设示范创建评估活 动更是有力推动了党中央关于法治政府建设决策部 署的贯彻落实。

\section{2. 坚持以人民为中心}

习总书记在中央全面依法治国工作会议上强调: “要坚持以人民为中心, 全面依法治国最广泛、最深 厚的基础是人民，必须坚持为了人民、依靠人民”。人 民当家作主是我国社会主义制度的核心, 全面推进依 法治国中的主体也必定是人民。人民群众作为与政府 机关联系最紧密、利益纠纷最频繁的群体, 在与政府 机关接触过程中也就最容易发现其存在的问题与不 足, 对于人民群众反应的问题、提出的建议与意见, 是法治政府改革所应当聚焦的重点。

法治政府建设的关键要素在于如何合理的约束、 规范公权力的使用, 进一步保障公民的权益。具体来 说, 就是要在行政立法、行政执法、行政争议解决等 具体行政行为之中尊重和保障老百姓的权利与利益。 近几年来, 国家修订了《行政诉讼法》, 提出对修改 《行政复议法》的意见等, 都体现出以人民为中心的 总体思路, 着力为人民群众打造更安全、更幸福、更 具获得感的法治政府。

\section{3. 坚持严格执法}

十八届四中全会中强调: “各级政府必须依法全 面履行职能, 坚持法定职责必须为、法无授权不可为, 健全依法决策机制, 完善执法程序, 严格执法责任, 做到严格规范公正文明执法。” 政府机关应牢记: “天 下之事, 不难于立法, 而难于法之必行”, 法律的活力 与权威性均来源于法律的实施, 如果没有实施法律, 或者实施地不到位, 那制定再多再完善的法律也无济 于事。行政机关作为有权实施行政行为的主体, 严格
执法是其基本的职能之一，也是联系群众最为紧密、 最为直接的职能, 更是推进依法治国的重要组成部分。 基于此, 严格执法是法治政府建设中的主要内容。倘 若不能严格执法, 则法治政府的建设便无从谈起。

党的十八届四中全会之后, 深化行政执法体制改 革越来越多的被人所提及, 加强执法活动的社会监督, 以及推行人性化执法、阳光执法、柔性执法等内容越 来越多的被实施, 有效降低了过去粗暴执法、野蛮执 法、一刀切式执法等问题, 人民群众的满意度逐年上 升。

\section{3. 大数据与法律的共性关系}

大数据最早运用在法学研究及法律事务工作中 主要是因为其所特有的海量信息处理能力, 能快速的 从大量数据中搜寻各类特定的目标信息, 极大地减轻 了法学学者以及实务工作者的搜索时间。随着大数据 的不断发展, 目前大数据所能给法学界与法律界带来 的不单单只有数据归整这一特定作用, 而是可以将不 同社会现象进行数字化处理, 并且通过不同种类的模 型分析、模拟社会实验等手段, 将纷繁复杂的社会现 象推导成某种初步的社会规律, 虽然这种初步的社会 规律仍需要使用者结合现实情况加以区分使用, 但是 这种新的研究方式可以帮助研究者们更快的把握对 事物, 尤其是新生事物的理解。现代科技的发展不仅 极大地推进了人类生产生活方式的改变, 更对于传统 的社会思维模式带来了新的认识角度。大数据与法律 间的关系是: “科学在本质上对人与自然实践关系的 根本表现”, 也是自然科学与社会科学相互融合, 并 与各类社会关系产生紧密联系的表象。

一方面, 法律作为人类逻辑体现的载体之一, 具 有强烈的客观现实性与主观意志性。数字技术作为自 然科学今后发展的重点, 其本质也具有着很强的逻辑 性, 但是这种逻辑性中人类意志的部分被大幅降低。 在这个背景下将大数据与法律进行融合存在着的主 要障碍是无法精确地对应匹配, 尤其是行政法这种应 用面广泛、权力间互相制约的法律。但在行政决策以 及法治政府评估等部分抽象行政行为中运用大数据 分析, 如果运用得当, 则可以突出大数据的快速分析 能力, 帮助行政机关选择最优的解决路径。

另一方面, 现阶段大数据虽然可以使用数字信号 模拟人类的思维模式, 但是其模拟方式以及模拟程度 仍远不及人类现有的思考水平, 加之受限于数据本身 存在的局限性以及分析结果在实际应用中所存在的 误差等问题, 大数据技术无法替代传统的分析决策过 程, 只是对分析决策起到辅助促进作用, 行政行为的 根本要求在于符合社会发展的基本需要。 


\section{4. 大数据参与行政决策的作用与路径}

\section{1. 大数据参与行政决策的作用}

行政决策是法治政府建设中重要环节, 也是诸多 行政行为的起点, 一个好的行政决策可以让行政行为 事半功倍。国务院于 2019 年颁布《重大行政决策程 序暂行条例》(以下简称暂行条例) 中明确强调: “作 出重大行政决策应当遵循科学决策原则...运用科学 技术和方法, 尊重客观规律, 适应经济社会发展和全 面深化改革要求。” 将大数据运用在行政决策, 尤其 是重大行政决策中也契合《暂行条例》的立法精神。

在大数据时代之前, 行政机关做出行政决策前所 收集到的数据来源庞多, 且收集方式大多以人工收集 为主, 具体方式有譬如实地调研、问卷调查、座谈宣 讲等方式。此种信息收集方式效率低下且容易出错, 而且可能因样本缺乏数据特征性等问题造成最终所 做出的决策与实际情况存在偏差。进入大数据时代后, 由于数据收集阶段所运用的收集手段的变化, 导致收 集数据的量成百倍增长，从而实现更多数据的占有。 而依靠大数据辅助决策最重要的就是前期收集到的 数据数量、数据种类、数据样式等所形成的数据集合。 加之数据分析软件的合理运用, 将所收集到的数据进 行可视化处理, 则能使行政机关更加直观的对数据进 行整理分析, 避免了仅依靠逻辑推理或主观臆断所造 成的不必要的错误决定。

除了上述大数据较传统信息收集方式有数据量 更庞大的特点外, 正确运用大数据的可带来的其他优 势还包括: 一、数据的高速处理能力。在拥有海量信 息数据的基础上, 大数据较传统数据处理方式更为高 效, 能够降低因信息处理不及时所导致的决策失时等 问题，提高政府机关的决策效率; 二、资源的整合利 用能力。过去政府机关各行政职能部门间除了成立联 动小组及综合办公室外, 信息交流往往只在自己系统 内部流通，同级部门间信息资源交换率低。大数据的 运用突破了这种隔阂，使得各部门间数据可以互通， 提高了资源的流动性，使得政府机关制定决策时可以 更加全面和完善的做出选择。

\section{2. 政府决策中大数据运用的路径构建}

政府决策中运用大数据技术应当从以下几方面 进行考虑: 第一, 数据来源应公开透明, 不得侵犯公 民的个人隐私。目前手机、平板、电脑等移动设备中 所使用的软件开发者为满足其了解顾客的消费能力、 消费喜好以及推销产品等目的, 允许软件自行收集使 用者的个人信息, 加之不法分子使用非法途径获取带 有个人身份的信息, 给软件使用者带来了非常多不必 要的麻烦。政府机关所做决策时，也会相应收集到很 多公民的信息, 其中一部分属于应当被保护的个人信 息, 例如姓名、年龄、身份证号码以及手机号码等, 政府机关应当对公民隐私权的保护有着积极的作为 义务。“大数据时代的隐私性主要体现在不暴露用户
敏感信息的前提下进行有效的数据挖掘, 这有别于传 统的信息安全领域更加关注文件的私密性等安全属 性。”政府在运用涉及公民隐私信息时，应当先征求 信息所有者的同意，对其 “去识别化” 后，再进行分 析使用。

应当建立决策追责机制, 约束行政机关滥用职权 的行为。法治政府建设中重要的环节在于如何科学的 约束行政机关的权力，防止权力的滥用。行政决策作 为大部分行政行为的 “起点”, 是行政权力运用的开 端, 自然应当受到约束, 而建立决策追责机制是解决 滥用职权的可行办法。

追责机制中应当注意区分追责主体，对于参与决 策制定的行政机关工作人员出现违反决策规定，导致 重大损失的情形时，可依据 “谁决策，谁负责” 的原 则，依据《中华人民共和国公职人员政务处分法》、

《中国共产党纪律处分条例》以及行政机关内部制定 的管理规定分别给予行政处分、党纪处分等。

对于在行政决策中起到辅助作用的技术人员、外 聘人员等造成决策失误的情形, 由于其身份不属于国 家公职人员，无法依据《中华人民共和国公职人员政 务处分法》对其进行政务处分，但是可依据其在决策 辅助中所起到的作用大小, 加之主观故意或过失, 追 究其民事责任甚至刑事责任。同时，还可以建立健全 “政务辅助失信名单”, 将故意或重大过失造成决策 失误的辅助人员列入 “失信名单” 并限制其与政府机 关进行业务往来，进一步降低此类行为发生的可能性。

\section{5. 大数据在法治政府建设评估中的作用与 路径}

大数据除了可以在政府决策中崭露头角外, 在法 治政府评估建设中也可以发挥其独到的作用。法治政 府建设评估是将管理学中有关评估原理、评估方式、 考核标准以及量化技术等方式方法与政府建设领域 进行交叉融合, 借助其他学科中有益的方式提升法治 政府的建设效果。

\section{1. 大数据应用于法治政府建设的作用}

将大数据应用在法治政府建设评估上有以下几 点优势：一、对现有评价方式进行优化。原有内部评 价方式同企业业绩考核相似, 带有较为强烈的绩效考 核的导向性，同时外部评价则需依靠第三方评估主体 辅助完成，但政府机关与第三方评估主体中存在有 “信息差”，第三方主体无法真实了解政府机关的实 际工作情况与完成情况，导致评估往往流于形式，无 法真正反映出政府建设中所存在的问题。通过引入大 数据技术，打破了原来存在于政府建设评估中的信息 “壁垒”，使得人们可以了解政府机关的工作进度与 完成情况，从侧面进一步推动了 “阳光政府”、“透明 政府” 的建设工作。同时使用大数据技术的同时还引 入了民间数据（社会团体调查、科研机构数据等）为 
官方数据做补充, 大大增强了评价内容的真实性与可 信度。二、可形成长期动态的观测机制。虽然法治政 府建设评估早在 2004 年前后就在我国开始实施, 但 由于近些年西方法治评估经验的引进对原有评估机 制造成的影响, 我国现有的法治政府建设评估并不完 善, 且诸如此类的政府建设评估需要有漫长的观察期, 故在观察过程中会产生大量的观察数据需要分析, 以 便寻找出需要的不足之处。若以传统方式对于海量数 据进行分析整理则会增加评估周期, 延缓良法善政作 出的时间。运用大数据技术则可以避免此类问题的产 生。通过大数据平台建设, 法治政府评估机构可以有 针对性地收集数据, 并按照年度做数据积累, 分别以时 间、地区、指标体系等维度构建研判线索轴, 实时比对 特定区域、特定选项不同待评指标, 长达数年的稳定、 全面的数据收集分析有利于形成长效的动态监测机 制。

\section{2. 大数据在法治政府建设中的路径构建}

要构建大数据技术在法治政府建设中的路径, 笔 者认为应当从以下角度进行构建: 一、加大大数据人 才培养力度, 助力评估综合建设。要将大数据技术妥 善运用在法治政府建设评估上需要从政府机关人才 培养与社会人才培养两方面着手。对于政府机关人才 培养, 需要从源头入手, 将大数据人才吸纳进公务员 队伍, 同时加强其政治与业务学习, 达到政治素质过 硬、业务水平合格、专业技能优秀的复合型公务员人 才, 同时加强政府工作人员对数据应用技能的培训, 提高对大数据收集、分析和运用的能力; 对于社会人 才培养而言, 需要高校、科研机构等发挥其学科优势 与人才优势, 培养与法治政府评估有关的大数据人才, 与政府需求挂钩。再者加大高新技术企业参与法治政 府建设评估的补占与优惠政策, 鼓励企业与政府机关 合作，为政府机关开发相应的评价系统。

搭建法治政府建设评估大数据信息平台。政府机 关运用大数据技术实现法治政府建设评估现代化需 要有信息平台作为支撑。首先, 应当明确建设目标, 信息平台的构建应当以法治政府建设的总目标为顶 层设计。由于不同地区发展程度不一, 在平台搭建过 程中, 首先应当在充分领会上级精神的前提下, 结合 地区发展态势综合研判决定需要建设包含何种信息 的平台, 其次在确定数据种类后应开始组织收集信息, 信息收集方式应当多样化，避免因数据收集手段单一 导致的数据量欠缺、样本缺乏特征性等问题, 再次需 要对数据进行归整、篮选、去识别化等操作, 减少人 为因素造成的信息失真。

\section{6. 结论}

在科学技术发展日新月异的今天, 将新技术运 用于法治政府建设当中符合国家的政策, 同时也对 政府建设起到了积极的推动作用。通过运用互联网 思维, 加强了企业和公众在政府建设中的社会参
与，从而形成协同治理的社会共识，打造政府治理 改革与社会多方需求充分对接的服务供给模式。实 现了政府各层级间的数据协调运用，还能发挥协调 区域间建设不平衡的矛盾、打破政府部门间数据壁 垒的功能, 成为打造协调发展治理格局的必要手 段，让法治政府建设中进程中完成自我更新。

随着 “大数据战略” 的不断推进, 目前已有部 分省份开始着手实施大数据助力政府改革且效果明 显。深入推进大数据在法治政府建设中的作用, 不 仅有利于增强政府机关的工作水平, 降低工作强 度, 提升社会治理能力, 更利于在信息化和智能化 的大数据发展浪潮中, 把握机遇, 助力实现中国 梦。

\section{REFERENCES}

[1] Shen,B.(2016) Foreign Policy Decision Process Supported by Big Data: Optimization and Limitations. International Forum,J,5:32-37+80.

[2] Meng X, Ci X,(2013) Big Data Management: Concepts, Technology and Challenges.Computer Research and Development,J,1:146-169.

[3] Chen L,Yang S, (2020) The application, problems and countermeasures of big data in the evaluation practice of government under the rule of law. Journal of Weinan Normal University. J.10: 82-86

[4] Ma H, (2020) On the theory of government by law in Xi Jinping's thought on the rule of law. Politics and Law Forum.J.38:11-22

[5] Qiu R (2015), In: Hao P. (Eds.) "Science and Technology Thought in the Theoretical System of Socialism with Chinese Characteristics", Jiangxi People's Publishing House, Jiangxi, 29-30

[6] Li R, (2020) Use new technology to accelerate the legalization of national governance. Chinese leadership science. J.5:84-86+124

[7] Wang Q, Yao W, He Aijun (2020) analyze the application of big data on the rule of law in smart justice. Chinese justice. J. 4:41-46

[8] Li L (2020) The application of big data in major administrative decision-making and its legalization. Journal of Chongqing University of Posts and Telecommunications (Social Science Edition). j.issn. 32(01),47-56

[9] Jiang B, Li S(2019) Tolerance and cooperation: the concept of administrative law of open government data in the era of big data. Hebei Law. j.cnki.37(12),101-109 